\title{
Spinal cord protection via alpha-2 agonist-mediated increase in glial cell-line-derived neurotrophic factor
}

\author{
Kirsten A. Freeman, MD, ${ }^{a}$ David A. Fullerton, MD, ${ }^{a}$ Lisa S. Foley, MD, ${ }^{a}$ Marshall T. Bell, MD, ${ }^{a}$ \\ Joseph C. Cleveland, Jr, MD, ${ }^{\mathrm{a}}$ Michael J. Weyant, MD, ${ }^{\mathrm{a}}$ Joshua Mares, BA, ${ }^{\mathrm{a}}$ Xianzhong Meng, PhD, MD, \\ Ferenc Puskas, MD, PhD, ${ }^{b}$ and $\mathrm{T}$. Brett Reece, $\mathrm{MD}^{\mathrm{a}}$
}

\begin{abstract}
Objectives: Delayed paraplegia secondary to ischemia-reperfusion injury is a devastating complication of thoracoabdominal aortic surgery. Alpha-2 agonists have been shown to attenuate ischemia-reperfusion injury, but the mechanism for protection has yet to be elucidated. A growing body of evidence suggests that astrocytes play a critical role in neuroprotection by release of neurotrophins. We hypothesize that alpha-2 agonism with dexmedetomidine increases glial cell-line-derived neurotrophic factor in spinal cord astrocytes to provide spinal cord protection.
\end{abstract}

Methods: Spinal cords were isolated en bloc from C57BL/6 mice, and primary spinal cord astrocytes and neurons were selected for and grown separately in culture. Astrocytes were treated with dexmedetomidine, and glial cell-line-derived neurotrophic factor was tested for by enzyme-linked immunosorbent assay. The 3-(4,5-dimethylthiazol-2-yl)-2,5-diphenyltetrazolium bromide assay was used to assess neuronal viability.

Results: Spinal cord primary astrocytes treated with dexmedetomidine at $1 \mu \mathrm{mol} /$ $\mathrm{L}$ and $10 \mu \mathrm{mol} / \mathrm{L}$ had significantly increased glial cell-line-derived neurotrophic factor production compared with control $(P<.05)$. Neurons subjected to oxygen glucose deprivation had significant preservation $(P<.05)$ of viability with use of dexmedetomidine-treated astrocyte media. Glial cell-line-derived neurotrophic factor neutralizing antibody eliminated the protective effects of the dexmedetomidine-treated astrocyte media $(P<.05)$.

Conclusions: Astrocytes have been shown to preserve neuronal viability via release of neurotrophic factors. Dexmedetomidine increases glial cell-derived neurotrophic factor from spinal cord astrocytes via the alpha-2 receptor. Treatment with alpha- 2 agonist dexmedetomidine may be a clinical tool for use in spinal cord protection in aortic surgery. ( $\mathrm{J}$ Thorac Cardiovasc Surg $2015 ; 149: 578-86)$

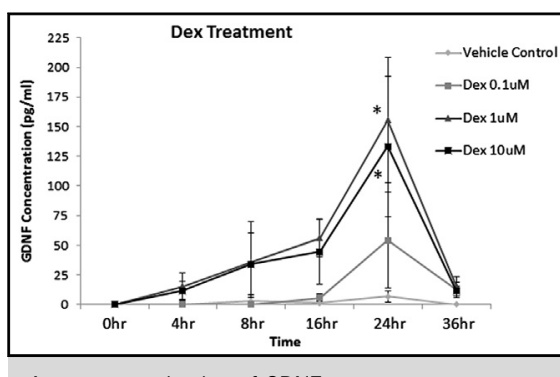

Astrocyte production of GDNF

\section{Central Message}

Alpha-2 agonism with dexmedetomidine protected the spinal cord from injury by increasing glial cell-derived neurotrophic factor in astrocytes.

\section{Clinical Relevance}

These in vitro studies propose a novel strategy to protect the spinal cord from injury. Dexmedetomidine, a clinically available sedative, is an agonist of neuronal $\alpha 2$-adrenergic receptors. This agent stimulated spinal cord astrocytes to increase glial cell-line-derived neurotrophic factor, a potent neuroprotective factor for motor neurons. This new approach may prevent paraplegia during aortic surgery.

See Editorial Commentary pages 586-7.
Delayed paraplegia secondary to ischemia-reperfusion (IR) injury remains a devastating complication of thoracoabdominal aortic surgery. Advances have been made in adjuvant protective techniques, including hypothermic circulatory

From the Departments of Surgery ${ }^{\mathrm{a}}$ and Anesthesiology, ${ }^{\mathrm{b}}$ University of Colorado Denver, Denver, Colo.

Funded by the Department of Surgery, University of Colorado Denver.

Disclosures: Authors have nothing to disclose with regard to commercial support.

Read at the 40th Annual Meeting of The Western Thoracic Surgical Association, Dana Point, California, June 25-28, 2014.

Received for publication June 26, 2014; revisions received Sept 22, 2014; accepted for publication Oct 4, 2014; available ahead of print Nov 21, 2014.

Address for reprints: Kirsten A. Freeman, MD, 12631 E 17th Ave, C310, Aurora, CO 80045 (E-mail: kirsten.freeman@ucdenver.edu).

$0022-5223 / \$ 36.00$

Copyright (c) 2015 by The American Association for Thoracic Surgery

http://dx.doi.org/10.1016/j.jtcvs.2014.10.037 arrest and other adjuncts, but paraplegia continues to be a postoperative setback. ${ }^{1}$ Ischemia and the inflammatory responses of reperfusion are both known to contribute to neuronal degeneration resulting in spinal cord dysfunction. ${ }^{2}$

Alpha-2 agonists have been shown to attenuate IR injury, but the mechanism for protection has yet to be elucidated. Cerebral, ${ }^{3,4}$ cardiac, ${ }^{5}$ lung, ${ }^{6}$ and renal ${ }^{7}$ models have all shown attenuation of IR injury by alpha- 2 agonists, and the highly selective alpha-2a agonist dexmedetomidine has been shown to provide functional attenuation of spinal cord IR injury in our in vivo murine model. ${ }^{8}$ The method by which dexmedetomidine protected the mice in our model has not been elucidated.

Astrocytes are the support cells of the central nervous system. Research has demonstrated that astrocytes play a 


\section{Abbreviations and Acronyms \\ ELISA $=$ enzyme-linked immunosorbent assay \\ $\mathrm{GDNF}=$ glial cell line-derived neurotrophic factor \\ IR $=$ ischemia-reperfusion \\ MTT = 3-(4,5-dimethylthiazol-2-yl)-2,5- \\ diphenyltetrazolium bromide \\ OGD = oxygen glucose deprivation \\ SEM $=$ standard error of the mean}

critical role in neuroprotection. ${ }^{9-12}$ In particular, they are critical in IR injury of neurons for a variety of reasons. Astrocytes are the most abundant cell type in the nervous system $^{13}$ with significant interconnections through gap junction channels. ${ }^{14}$ They play a vital role in coupling neuronal activity blood flow that underlies the hemodynamic responses. ${ }^{15}$ Astrocytes are also central to neuroprotection by release of neurotrophins. ${ }^{16-18}$

Specifically, astrocytes are the major source of a glial cell line-derived neurotrophic factor (GDNF). ${ }^{19}$ GDNF has been shown to be a potent neurotrophic factor for motor neurons and to modulate neuronal death, ${ }^{20}$ as well as to support neuronal survival and regeneration. ${ }^{21}$ GDNF has yet to be examined in spinal cord IR injury; however, GDNF has shown functional recovery in a model of traumatic spinal cord injury. ${ }^{22}$ An ongoing question is how to enhance the astrocyte production of GDNF. Significantly, the alpha- 2 agonist dexmedetomidine has been linked to increased production of $\mathrm{GDNF}^{23} \mathrm{We}$ hypothesize that alpha-2 agonism with dexmedetomidine increases GDNF production in spinal cord astrocytes to provide spinal cord protection after IR injury.

\section{MATERIALS AND METHODS Materials}

Dexmedetomidine and atipamezole were purchased from Tocris Bioscience (Ellisville, Mo). Anti-GDNF neutralizing antibody was purchased from Abcam (Cambridge, Mass).

\section{Animals \\ The Animal Care and Use Committee at the University of Colorado at Denver Health Sciences Center approved all experiments. This investigation conformed to the Guide for the Care and Use of Laboratory Animals (US National Institutes of Health publication No. 85-23, National Academy Press, Washington, DC, revised 1996). C57BL/6 mice aged postnatal day 2 to 3 from Jackson Laboratories (Bar Harbor, Maine) were used for all experiments. Each litter in total was considered $n=1$.}

\section{Cell Culture}

Primary spinal cord neuron cultures and primary astrocyte cultures were obtained from mice aged 2 to 3 days. Briefly, the mice were euthanized with isoflurane and then decapitated. The vertebral column was dissected out, and the spinal cord was removed en bloc via injection of cold phosphatebuffered saline ( $\mathrm{pH}$ 7.4) through the spinal canal. The spinal cord tissue was minced and then digested in a solution of Hibernate-A (Invitrogen, Carlsbad, Calif) with Papain (Worthington, Lakewood, NJ). Neurons and astrocytes were isolated using an OptiPrep (Sigma-Aldrich, St Louis, Mo) density gradient adapted from Brewer and Torricelli. ${ }^{24}$

Neurons were plated on plates coated with Poly-D-Lysine (Sigma-Aldrich) at approximately 300,000 cells/well on a 24-well plate in $1 \mathrm{~mL}$ culture media of Neurobasal-A (Invitrogen), B27 (Invitrogen), GlutaMAX (Invitrogen), and penicillin/streptomycin (Gibco, New York, NY). On in vitro day 3, AraC (Sigma-Aldrich) was added to prevent astrocyte replication. Cell cultures were maintained in a humidified atmosphere containing $5 \% \mathrm{CO}_{2}$ at $37^{\circ} \mathrm{C}$ and underwent half media change every 3 days. The cultures were greater than $90 \%$ neurons as seen by morphology on light microscope and confirmed with microtubule-associated protein 2-positive neuronal staining. Cells were used at in vitro days 7 to 10 for experimentation, which is considered mature for neuronal cultures.

Astrocytes were placed in culture flasks or plated on 12-well plates in culture media of Neurobasal-A (Invitrogen), B27 (Invitrogen), GlutaMAX (Invitrogen), fetal bovine serum (Gibco), and penicillin/streptomycin (Gibco). Astrocytes were maintained in culture and allowed to grow to confluence before experimentation. Astrocyte medium used for experimentation was collected and stored in a $-80^{\circ} \mathrm{C}$ refrigerator until use.

\section{Oxygen Glucose Deprivation}

On the day of the experiment, the experimental medium of Dulbecco's Modified Eagle's Medium without glucose (Gibco) was placed in the Ruskinn Bug Box Plus (Ruskinn Technology Ltd, Bridgend, South Wales, UK) humidified airtight hypoxic chamber for 2 hours. The Ruskinn Bug Box Plus was used per the manufacturer's protocol to maintain an environment of $95 \% \mathrm{~N}_{2} / 5 \% \mathrm{CO}_{2}$ at $37^{\circ} \mathrm{C}$. A hypoxic environment was verified with Anaerobic Indicator Strips (Oxoid Ltd, Basingstoke, Hants, UK) before placement of media in the chamber, and hypoxia of the media after 2 hours was tested with an indicator strip. The neuronal maintenance culture medium was then removed, the cells were washed with phosphate-buffered saline, and $1 \mathrm{~mL}$ of glucose-deprived medium previously treated in the hypoxic chamber was added to each of the cell culture wells on a 24-well plate. Oxygen glucose deprivation (OGD) was induced by placing the plates in the hypoxic chamber for the experimental time periods. A hypoxic environment in the chamber was tested with an indicator strip before placement of cells into the chamber and on the completion of experimentation time to confirm a hypoxic environment. After OGD was completed, neurons were returned to a normal incubator for reperfusion and OGD media were replaced with normal maintenance neuronal media versus experimental astrocyte media.

\section{Viability Studies}

For viability studies, neuronal cells were cultured on 24-well plates. Cell viability was determined with the MTT Cell Proliferation Kit (Roche Diagnostics, Indianapolis, Ind) according to the procedure provided by the manufacturer. Briefly, 3-(4,5-dimethylthiazol-2-yl)-2,5diphenyltetrazolium bromide (MTT) was added to each well at a concentration of $0.5 \mathrm{mg} / \mathrm{mL}$ and incubated for 4 hours at $37^{\circ} \mathrm{C}$, and then dimethyl sulfoxide solution was added to the wells. The absorbance at $630 \mathrm{~nm}$ was measured on a BioTek Synergy H1 Hybrid microplate reader (BioTek Instruments Inc, Winooski, Vt). Cell viability is presented as the percentage of absorbance relative to nonischemic control.

\section{Enzyme-Linked Immunosorbent Assay}

After the experiments were conducted, cell culture supernatants were collected. A standard enzyme-linked immunosorbent assay (ELISA) was performed using these supernatants. A GDNF (Abcam) ELISA was performed according to the procedure provided by the manufacturer, and the absorbance at $450 \mathrm{~nm}$ was measured on a BioTek microplate reader. Data are presented as concentration in mean picograms/milliliter \pm standard error of the mean. 

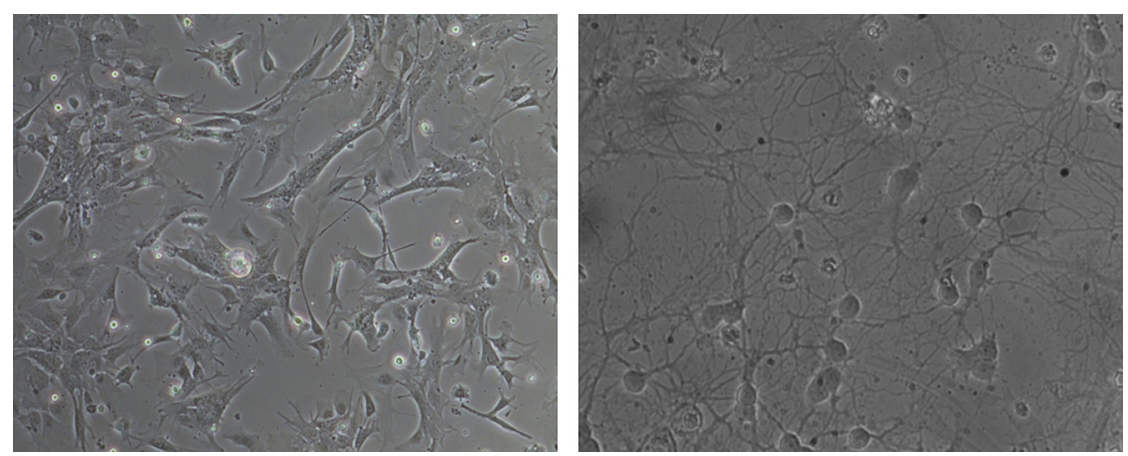

FIGURE 1. Light microscopy image of primary spinal cord astrocytes (left) and primary spinal cord neurons (right) at $100 \times$ magnification.

\section{Statistics}

Results are presented as mean \pm standard error. Data were analyzed with StatView software (SAS Institute Inc, Cary, NC). Statistical analysis was performed using 1-way analysis of variance and Student $t$ test.

\section{RESULTS}

\section{Spinal Cord Cell Cultures}

By using our spinal cord isolation protocol, we were successfully able to culture and maintain primary cultures of spinal cord astrocytes and neurons as seen by the light microscopy image in Figure 1. Astrocytes are identified by light microscope by their star-shaped morphology. Astrocytes are robust cells in culture and grow to confluence easily, whereas neurons are more fragile cells in culture. Neurons are likewise identifiable by morphology on light microscope by their cell bodies and projections. Mature neurons in culture will develop a network of projections as shown in Figure 1.

\section{Glial Cell Line-Derived Neurotrophic Factor Production}

When primary spinal cord astrocytes were treated with dexmedetomidine, we found that GDNF concentration increased in a dose-dependent and time-dependent fashion (Figure 2). GDNF was measured with a GDNF ELISA kit at varying time points after treatment with dexmedetomidine. The concentration of dexmedetomidine used was 0.1 $\mu \mathrm{mol} / \mathrm{L}, 1 \mu \mathrm{mol} / \mathrm{L}$, and $10 \mu \mathrm{mol} / \mathrm{L}$. Although not significant, there was an increase in GDNF concentration at 0.1 $\mu \mathrm{mol} / \mathrm{L}$ at 16 hours, 24 hours, and 36 hours. However, there was a significant increase in GDNF production at 24 hours with dexmedetomidine at $1 \mu \mathrm{mol} / \mathrm{L}$ and $10 \mu \mathrm{mol} / \mathrm{L}$ compared with vehicle control $(P<.05)$. The GDNF level appears to peak at 24 hours, given the levels at 36 hours were lower, indicating the GDNF is degraded or used by the cells between the 24- and 36-hour timepoints.

There was not a significant difference between $1 \mu \mathrm{mol} / \mathrm{L}$ and $10 \mu \mathrm{mol} / \mathrm{L}$ treatment when compared with each other. We also found an increase in GDNF production with 1 $\mu \mathrm{mol} / \mathrm{L}$ and $10 \mu \mathrm{mol} / \mathrm{L}$ treatment at 4 hours, 8 hours, 16 hours, and 36 hours, but these increases were not statistically significant. On the basis of these data, we decided to use astrocyte-conditioned medium at 24 hours post-treatment with $1 \mu \mathrm{mol} / \mathrm{L}$ of dexmedetomidine for further studies to determine whether GDNF preserves the viability of neurons injured by IR.
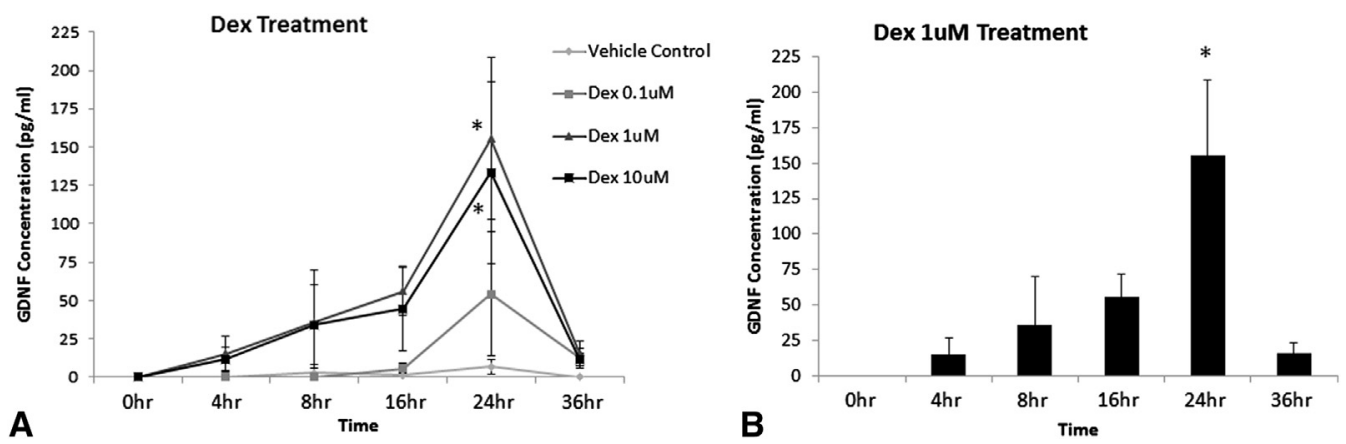

FIGURE 2. Astrocyte production of GDNF with dexmedetomidine treatment. A, There was a significant increase at $24 \mathrm{hours}$ with $1 \mu \mathrm{mol} / \mathrm{L}$ and $10 \mu \mathrm{mol} / \mathrm{L}$ of dexmedetomidine compared with vehicle control $(* P<.05)$. Data are presented as mean GDNF concentration in $\mathrm{pg} / \mathrm{mL} \pm \mathrm{standard}$ error of the mean $(\mathrm{SEM}), \mathrm{n}=4$. $\mathrm{B}$, There is a significant increase in GDNF production at 24 hours compared with other time points with dexmedetomidine treatment of 1 $\mu \mathrm{mol} / \mathrm{L}\left({ }^{*} P<.05\right)$. Data are presented as mean GDNF concentration in $\mathrm{pg} / \mathrm{mL} \pm \mathrm{SEM}, \mathrm{n}=4$. Dex, Dexmedetomidine; GDNF, glial cell line-derived neurotrophic factor. 


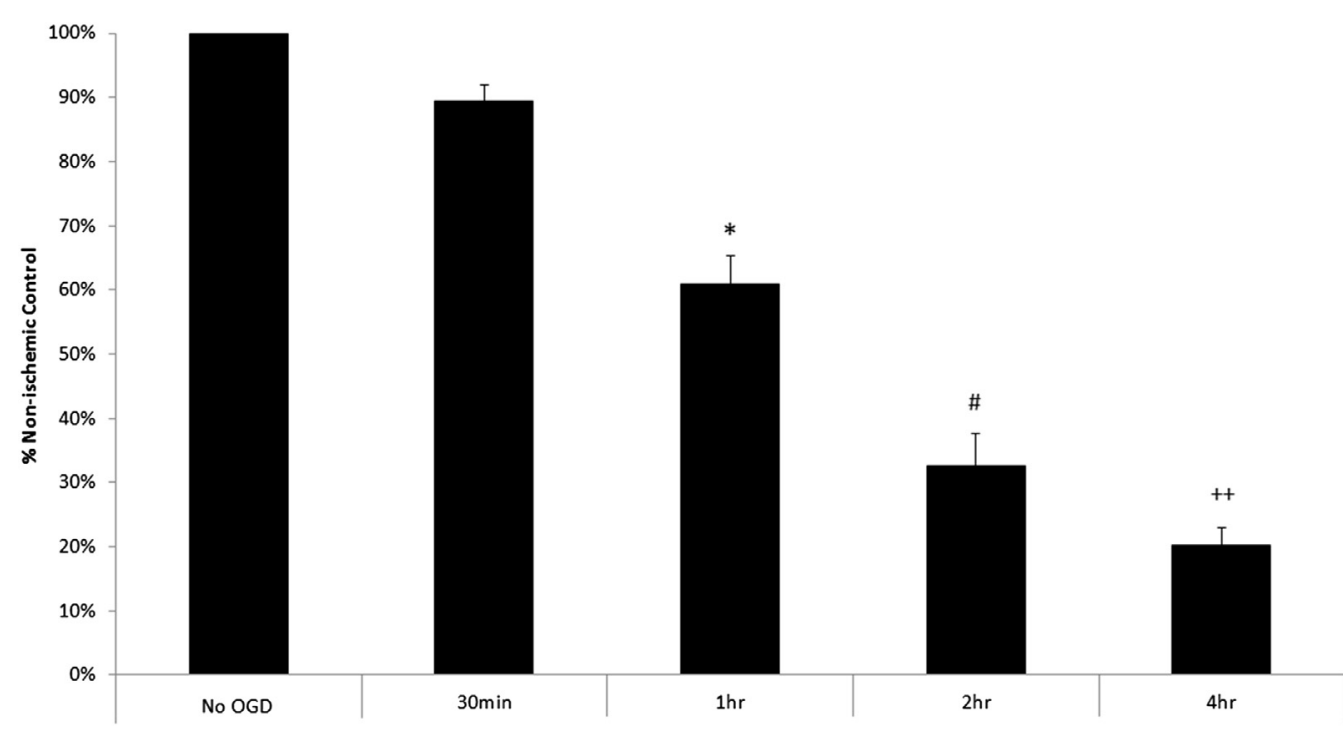

FIGURE 3. Ischemic dose-response curve of neurons subjected to OGD. At 1 hour, there is a significant decrease in viability $(* P<.05)$ and a further significant decrease at 2 hours $(\# P<.05)$ and 4 hours $(++P<.05)$. There was a decrease, but not statistically significant, at 30 minutes. Viability determined by MTT assay and presented as mean percent control $\pm \mathrm{SEM}, \mathrm{N}=5$. $O G D$, Oxygen glucose deprivation.

\section{Neuronal Viability With Oxygen Glucose Deprivation}

We then wanted to determine whether GDNF produced in the astrocyte medium could salvage neurons injured by IR injury. OGD was used as our model for IR injury. We used 30 minutes, 1 hour, 2 hours, and 4 hours of ischemia time followed by 24 hours of reperfusion to determine an ischemic dose-response curve for neuronal viability as assessed by MTT assay (Figure 3). We found that there was not a significant decrease in neuronal viability at 30 minutes of OGD ( $90 \%$ viable), but that there was a significant decrease in neuronal viability at 1 hour of OGD to only $61 \%$ viable $(P<.05)$. In addition, there was a further significant decrease at 2 hours $(P<.05)$ and 4 hours of OGD $(P<.05)$ with $33 \%$ and $20 \%$ viable, respectively. One hour of OGD was chosen for further studies given that there was a significant decrease in neuronal viability, but enough neurons remained viable for further experimentation.

\section{Neuronal Viability With Dexmedetomidine-Treated Astrocyte Media}

Astrocyte-conditioned media, dexmedetomidine-treated astrocyte media, are defined here as astrocyte media that are 24 hours post-treatment with $1 \mu \mathrm{mol} / \mathrm{L}$ of dexmedetomidine, which has the maximal amount of GDNF (Figure 2). The dexmedetomidine-treated astrocyte media were then placed on neurons that had been subjected to OGD in replacement of the normal neuron media that would be used during reperfusion time. Viability was assessed with the use of an MTT assay. As anticipated, the results show that at 1 hour of OGD, the viability of the neurons is significantly decreased $(P<.05)$ to approximately $62 \%$. We also found that with 1 hour of OGD there was a significant preservation in viability to $77 \%(P<.05)$ with the dexmedetomidine-treated astrocyte media compared with standard neuron reperfusion media (Figure 4). The alpha2 inhibitor atipamezole was given to all the neuronal cells to prevent direct effects of residual dexmedetomidine in the astrocyte-conditioned media.

\section{Glial Cell Line-Derived Neurotrophic Factor Preservation of Neuronal Viability}

To determine whether the preservation in viability that was seen from the dexmedetomidine-treated astrocyte media was due to GDNF, we used a neutralizing GDNF antibody to block the effects of GDNF on the neurons. By using the GDNF neutralizing antibody, we found that neurons had a significant loss in viability down to $66 \%$ compared with the $77 \%$ viable neurons given dexmedetomidine-treated astrocyte media without the neutralizing antibody $(P<.05)$ (Figure 5). This illustrates that GDNF is an important factor in neuronal survival after IR injury.

There was no statistical significance between the control reperfusion media and the dexmedetomidinetreated astrocyte media with GDNF neutralizing antibody; however, there was an increased viability with the dexmedetomidine-treated astrocyte media with neutralizing antibody to $66 \%$ compared with the control media at $62 \%$. This means that neurons did not lose all the preservation in viability that the astrocyte-conditioned medium provided when the GDNF neutralizing antibody was used, suggesting that there may be other attributes in the 


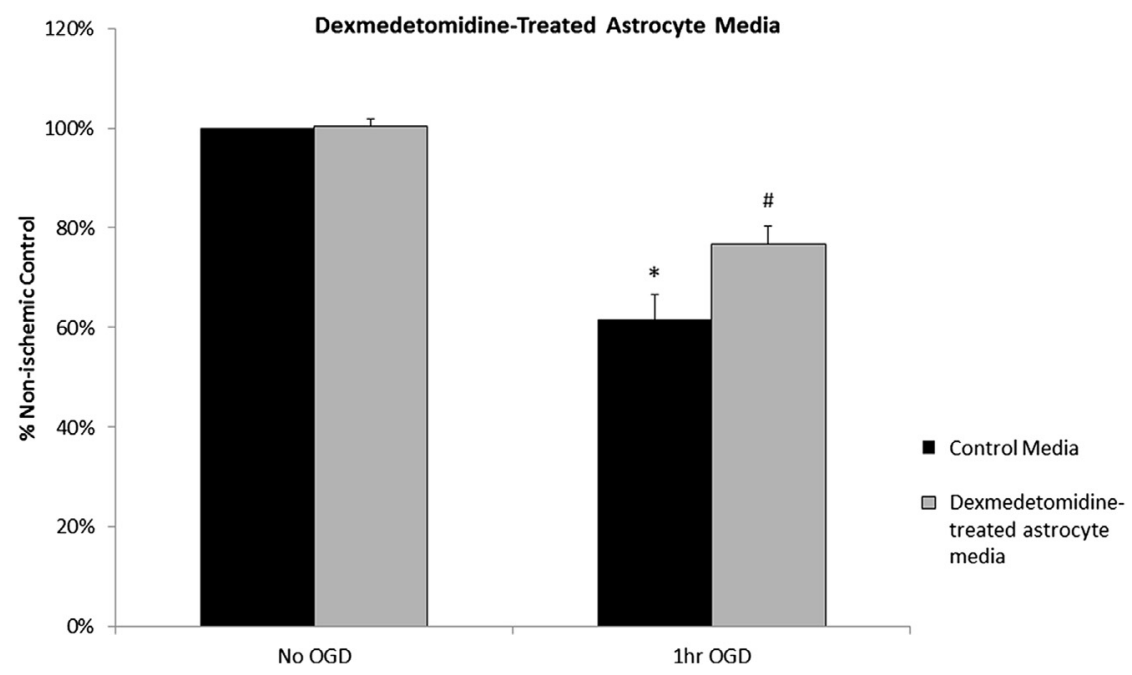

FIGURE 4. Effects of astrocyte-conditioned media on neuronal viability. There is a significant decrease in neuronal viability down to $62 \%$ at 1 hour OGD with control media compared with no OGD $\left({ }^{*} P<.05\right)$. With 1 hour of OGD, there is a significant increase in neuronal viability to $77 \%$ with dexmedetomidine-treated astrocyte media $(\# P<.05)$ compared with control neuron media. Results presented as mean percent nonischemic control \pm $\mathrm{SEM}, \mathrm{N}=8$. $O G D$, Oxygen glucose deprivation.

dexmedetomidine-treated astrocyte media that is also providing protection independently of GDNF.

\section{DISCUSSION}

Delayed paraplegia is a devastating complication of thoracoabdominal aortic surgery, which is secondary to IR injury. Alpha-2 agonists, including dexmedetomidine, have been shown to attenuate IR injury in many organ systems; however, the mechanism continues to not be well

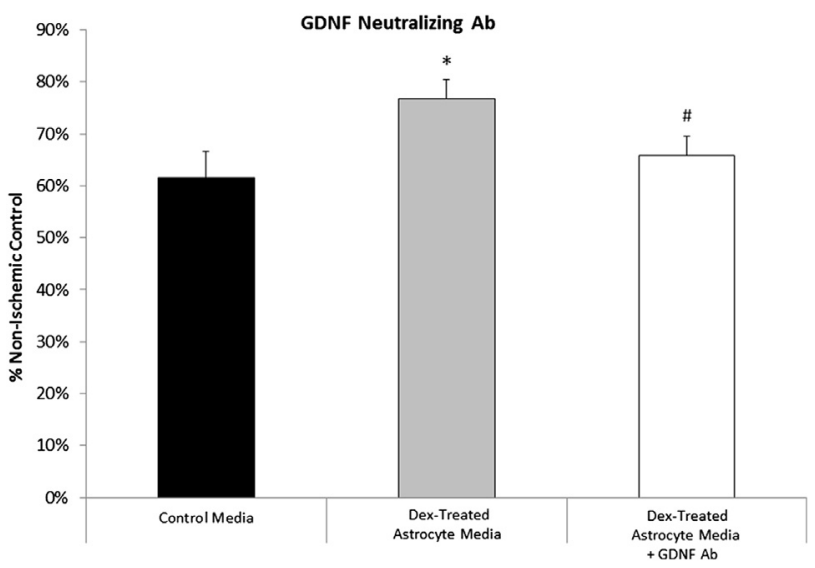

FIGURE 5. GDNF preserves neuronal viability. With 1 hour of OGD, there is an increase in neuronal viability with astrocyte-conditioned media to $77 \%$ compared with $62 \%$ with control reperfusion media $(* P<.05)$. The GDNF neutralizing antibody produced a significant loss in viability down to $66 \%$ compared with those given astrocyte-conditioned medium without the neutralizing antibody $(\# P<.05)$. There was no statistical significance between the astrocyte-conditioned media with neutralizing antibody and the control media. Results presented as mean percent nonischemic control $\pm \mathrm{SEM}, \mathrm{N}=8$. Ab, Antibody; $G D N F$, glial cell line-derived neurotrophic factor. understood. Our laboratory has primarily focused on spinal cord IR injury and delayed paraplegia. ${ }^{8,25,26}$ Through our in vivo murine model, we have further explored the treatment benefits and mechanisms of dexmedetomidine, although there are limitations to the in vivo model.

The limitations demonstrated in the in vivo murine model are multiple. First, the spinal cord can be treated only as a single entity. More clearly, the injection of dexmedetomidine affects every cell in the spinal cord. Once treatment has been completed and the mice are sacrificed, the entire spinal cord homogenate is studied with all cellular components included. With the in vitro model developed for this study, primary spinal cord neurons and primary spinal cord astrocytes can be cultured separately. Therefore, the effects of treatment to individual cell lines can be studied in isolation. We then are able to study the subsequent effects on each cell type independently in addition to the effects of substance released by those cell lines on one another.

Astrocytes were formerly thought to play minimal role in neuroprotection; however, an ever increasing body of literature suggests that they may be more critical to neuronal preservation. ${ }^{9,11,12}$ Astrocytes are the most abundant cell type and have been shown to play key roles in both normal and pathologic central nervous functioning. Structurally, astrocytes organize the architecture of the central nervous system and help organize communication pathways. ${ }^{13}$ Astrocytes are also the principal housekeeping cells of the nervous system, functioning in supportive tasks to control ion and water homeostasis, release neurotrophic factors, shuttle metabolite and waste products, and participate in the formation of the blood-brain barrier. ${ }^{10}$ Failure of these support mechanisms can be detrimental to neuronal survival, particularly in an injured state. 
Astrocytes are known to be a major source of GDNF. ${ }^{19}$ GDNF is a potent neurotrophic factor for motor neurons. ${ }^{20}$ It has also been shown to support neuronal survival and regeneration, ${ }^{21}$ and has been a major source of research in the Parkinson's literature for motor neuron recovery. ${ }^{16-18}$ Specifically crucial to our model is that GDNF production has been linked to alpha-2 agonism in cerebral models. ${ }^{23}$

We hypothesized that alpha- 2 agonism with dexmedetomidine increases GDNF in spinal cord astrocytes to provide spinal cord protection against neurons subjected to IR injury. The results of our study indicate that primary spinal cord astrocytes grown in vitro significantly produce GDNF with alpha-2 stimulation with $1 \mu \mathrm{mol} / \mathrm{L}$ and $10 \mu \mathrm{mol} / \mathrm{L}$ of dexmedetomidine. Our data suggest that GDNF is in its highest concentration after 24 hours of treatment. GDNF appears to be degraded or used by the cells between 24 and 36 hours given the decline in GDNF seen at 36 hours. There are significant clinical implications to the GDNF peak that we have observed in our experiments. It suggests that administration of dexmedetomidine in the clinical setting would likely need to be 1 day before the planned surgery to maximize GDNF concentration in the spinal cord at the time of IR injury.

The data show that dexmedetomidine-treated astrocyte media with high levels of GDNF are neuroprotective for neurons subjected to OGD. OGD is our in vitro model for IR injury. We accomplish OGD by treating the cell with glucose-free media and placing the cells in a hypoxic chamber to mimic ischemia followed by replacement with maintenance media with glucose and placement back into a normal incubator to simulate reperfusion. This model uses an experimental media free of glucose and oxygen media, which is crucial to our ischemic model given that clinically ischemia deprives cells of both their source of oxygen and glucose, thereby separating it from a simple hypoxia-reoxygenation model.

After 1 hour of OGD, only approximately $60 \%$ of neurons remain viable after 24 hours of reperfusion. With dexmedetomidine-treated astrocyte media during reperfusion, the neuronal viability is significantly increased to $77 \%$ viability. This study also found that use of a GDNF neutralizing antibody abolishes the majority of the protective effects provided by the dexmedetomidinetreated astrocyte media, suggesting that the majority of the effect on viability is due to neuroprotection by GDNF.

\section{Study Limitations}

No study is without limitations, especially an in vitro study meant to model a clinical scenario of IR injury seen in delayed paraplegia. In vitro dosing is difficult to translate to human subjects, but our previous in vivo model indicates that dosing can be appropriately increased to see treatment effects in a murine model and likely could be translated into human subjects without difficulty. Further, in vitro studies are crucial for studying individual cells but do not allow for the study of the cells within their normal microenvironment, and thus may limit understanding of treatment effects. Additional studies of astrocyte-conditioned medium are also needed to determine whether there are other properties that may provide neuroprotection in addition to GDNF. In particular, another future direction for our studies should involve co-culturing neurons and astrocytes to further mimic the true microenvironment the motor neurons are surrounded by in the spinal cord.

Another specific limitation of our in vitro study is the need to use perinatal mice for culturing neurons, as well as the inability to separate motor from sensory neurons in the spinal cord. A hallmark of in vitro cell culture is that cells need to appropriately survive to allow experimentations to be done. Surviving in culture can even be a struggle for neurons isolated from perinatal mice, but adult neuron cultures are so unpredictable in their ability to survive, studies of them are difficult to interpret, particularly the experimentations designed to detect changes in viability that we were studying. Therefore, we use a perinatal model to have predictable cell survival in culture. Also, there is an inability to separate motor from sensory neurons in our culture. Therefore, we do not know precisely which cell populations we are salvaging with dexmedetomidine; however, we do know that GDNF has been shown to specifically rescue motor neurons. ${ }^{17,18,21,22}$ Therefore, there is a possibility that one cell type is being preferentially saved over the other; however, our perinatal neuronal culture results mirror the functional outcomes found in our in vivo adult mouse model, and thus we believe our in vitro model is a good representation of spinal cord neuronal cell death of motor neurons that account for paraplegia seen in our adult murine in vivo studies.

\section{CONCLUSIONS}

Our data suggest that astrocytes and GDNF play an important role in neuroprotection from IR injury and may provide a strategy for protection of the spinal cord during aortic surgery.

The authors thank the Department of Surgery, University of Colorado Denver, for financial support for this project.

\section{References}

1. Conrad MF, Ye JY, Chung TK, Davison JK, Cambria RP. Spinal cord complications after thoracic aortic surgery: long-term survival and functional status varies with deficit severity. J Vasc Surg. 2008;48:47-53.

2. Stirling DP, Yong VW. Dynamics of the inflammatory response after murine spinal cord injury revealed by flow cytometry. J Neurosci Res. 2008;86:1944-58.

3. Zhang Y, Kimelberg H. Neuroprotection by Alpha-2 adrenergic agonists in cerebral ischemia. Curr Neuropharmacol. 2005;3:317-23.

4. Ma D, Hossain M, Rajakumaraswamy N, Arshad M, Sanders RD, Franks NP, et al. Dexmedetomidine produces its neuroprotective effect via the alpha 2A-adrenoceptor subtype. Eur J Pharmacol. 2004;502:87-97. 
5. Ibacache M, Sanchez G, Pedrozo Z, Galvez F, Humeres C, Echevarria G, et al. Dexmedetomidine preconditioning activates pro-survival kinases and attenuates regional ischemia/reperfusion injury in rat heart. Biochim Biophys Acta. 2012; 1822:537-45.

6. Gu J, Chen J, Xia P, Tao G, Zhao H, Ma D. Dexmedetomidine attenuates remote lung injury induced by renal ischemia-reperfusion in mice. Acta Anaesthesiol Scand. 2011;55:1272-8.

7. Gu J, Sun P, Zhao H, Watts HR, Sanders RD, Terrando N, et al. Dexmedetomidine provides renoprotection against ischemia-reperfusion injury in mice. Crit Care. 2011;15:R153.

8. Bell MT, Puskas F, Smith PD, Agoston VA, Fullerton DA, Meng X, et al. Attenuation of spinal cord ischemia-reperfusion injury by specific $\alpha$-2a receptor activation with dexmedetomidine. J Vasc Surg. 2012;56:1398-402.

9. Barreto GE, Gonzalez J, Torres Y, Morales L. Astrocytic-neuronal crosstalk: implications for neuroprotection from brain injury. Neurosci Res. 2011;71:107-13.

10. Takano T, Oberheim NA, Cotrina ML, Nedergaard M. Astrocytes and ischemic injury. Stroke. 2009;40(Suppl 1):S8-12.

11. Panickar KS, Norenberg MD. Astrocytes in cerebral ischemic injury: morphological and general considerations. Glia. 2005;50:287-98.

12. Bekker A, Sturaitis MK. Dexmedetomidine for neurological surgery. Neurosurgery. 2005;57(1 suppl):1-10.

13. Barreto G, White RE, Ouyang Y, Xu L, Giffard RG. Astrocytes: targets for neuroprotection in stroke. Cent Nerv Syst Agents Med Chem. 2011;11:164-73.

14. Giaume C, Koulakoff A, Roux L, Holcman D, Rouach N. Astroglial networks: a step further in neuroglial and gliovascular interactions. Nat Rev Neurosci. 2010; 11:87-99.

15. Arai K, Lo EH. Astrocytes protect oligodendrocyte precursor cells via MEK/ERK and PI3K/Akt signaling. J Neurosci Res. 2010;88:758-63.

16. Migliore MM, Ortiz R, Dye S, Campbell RB, Amiji MM, Waszczak BL. Neurotrophic and neuroprotective efficacy of intranasal GDNF in a rat model of Parkinson's disease. Neuroscience. 2014;274:11-23.

17. Nam JH, Leem E, Jeon MT, Jeong KH, Park JW, Jung UJ, et al. Induction of GDNF and BDNF by hRheb(S16H) transduction of SNpc neurons: neuroprotective mechanisms of hRheb( $\mathrm{S} 16 \mathrm{H})$ in a model of Parkinson's disease. Mol Neurobiol. May 25, 2014 [Epub ahead of print].

18. Akerud P, Canals JM, Snyder EY, Arenas E. Neuroprotection through delivery of glial cell line-derived neurotrophic factor by neural stem cells in a mouse model of Parkinson's disease. J Neurosci. 2001;21:8108-18.

19. Trendelenburg G, Dirnagl U. Neuroprotective role of astrocytes in cerebral ischemia: focus on ischemic preconditioning. Glia. 2005;51:307-20.

20. Nicole O, Ali C, Docagne F, Plawinski L, MacKenzie ET, Vivien D, et al. Neuroprotection mediated by glial cell line-derived neurotrophic factor: involvement of a reduction of NMDA-induced calcium influx by the mitogenactivated protein kinase pathway. J Neurosci. 2001;21:3024-33.

21. Pajenda G, Hercher D, Marton G, Pajer K, Feichtinger G, Maleth J, et al. Spatiotemporally limited BDNF and GDNF overexpression rescues motoneurons destined to die and induces elongative axon growth. Exp Neurol. 2014;261: 367-76.

22. Ansorena E, De Berdt P, Ucakar B, Simon-Yarza T, Jacobs D, Schakman O, et al. Injectable alginate hydrogel loaded with GDNF promotes functional recovery in a hemisection model of spinal cord injury. Int J Pharm. 2013; 455:148-58.

23. Yan M, Dai H, Ding T, Dai A, Zhang F, Yu L, et al. Effects of dexmedetomidine on the release of glial cell line-derived neurotrophic factor from rat astrocyte cells. Neurochem Int. 2011;58:549-57.

24. Brewer G, Torricelli J. Isolation and culture of adult neurons and neurospheres. Nat Protoc. 2007;2:1490-8.

25. Smith PD, Puskas F, Meng X, Lee JH, Cleveland JC Jr, Weyant MJ, et al. The evolution of chemokine release supports a bimodal mechanism of spinal cord ischemia and reperfusion. Circulation. 2012;126(11 Suppl 1):S110-7.

26. Smith PD, Puskas F, Meng X, Cho D, Cleveland JC Jr, Weyant MJ, et al. Ischemic dose-response in the spinal cord: both immediate and delayed paraplegia. J Surg Res. 2012;174:238-44.

\section{Discussion}

Dr Freeman. I'd like to thank the Western Thoracic Surgical Association, our moderators, and the members and guests. Today I'm going to talk with you about spinal cord IR injury.
As we know, paraplegia continues to be a devastating complication in thoracoabdominal aortic interventions, particularly in high-risk patients. We know that spinal cord ischemia then triggers resultant metabolic derangements that lead to a reperfusion injury. The alpha- 2 agonist dexmedetomidine, which we know better Precedex (Hospira, Lake Forest, Ill), has been shown to attenuate IR injury in a variety of organ systems. In an in vivo mouse model, we previously showed that pretreatment with dexmedetomidine preserved functional outcome after the spinal cord IR injury. However, as with any model, this in vivo model had its limitations, particularly with having to use the whole spinal cord. We were unable to separate the effects that the dexmedetomidine had on the specific cellular components of the spinal cord. So the mechanism has yet to be fully elucidated.

Our original aims were to first maintain spinal cord cell cultures of astrocytes and neurons, and then subject the cells to an in vitro model of IR injury, called "OGD." Our model of cell culture begins by harvesting cells from a spinal cord in block from postnatal mice. You may wonder why we have to use postnatal mice versus adult mice, and it's simply because adult mice neurons do not grow in culture. Then you follow by a digestion of the tissue and select it for a specific cell type with OptiPrep (Sigma-Aldrich, St Louis, Mo). What we are able to do further is select for neurons by the way you plate them and the type of media they are placed in. After they are in culture for 7 to 10 days, they are considered a mature neuron culture.

Astrocytes are likewise plated and grown in a media, basically the same as a neuron media but with addition of serum. This is a light microscope image of our neurons, and these are again considered mature. These are approximately 7 days in vitro, and you can see all the projections between each neuron. These are astrocyte cultures, and you can see by their morphology that they are different from the neurons.

Our model of OGD for IR injury begins by placing the oxygen glucose-deprived media onto the cells and then placing those cells into an anoxic chamber to simulate ischemia. Then we return the cells to a normal incubator with a normal neuron media to simulate a reperfusion.

What we found is basically an ischemic dose response with the OGD of neurons. At 30 minutes of OGD, neurons had a decrease in their viability. However, this decrease wasn't statistically significant. At 1 hour, we went down to a viability of neurons at approximately $60 \%$, and this was statistically significant. At a further 2 and 4 hours, we had a statistical decrease in viability. We chose the $60 \%$ viable, the 1-hour OGD, for further studies for 2 reasons. There are enough cells remaining that you can potentially do further studies on them, and at that time point we had a statistical decrease in viability.

What we wanted to do after we were able to culture the cells, and then after we were able to kill the cells, is to find a way to preserve the cells. One way that we were interested in looking at doing this is the astrocytes around the neurons. In vitro neurons are by themselves; however, in the spinal cord, they are surrounded by astrocytes. Astrocytes are the most abundant cell type in the nervous system. They comprise approximately $85 \%$ of the cells. They organize the architecture, provide communication pathways, control ion homeostasis, and participate in the blood-brain barrier. Also, 
failure of these support systems can be detrimental to neuron survival in general, but specifically in an injured state.

Astrocytes are thought to provide specific neuroprotection by release of neurotrophic factors, and astrocytes are the major source of GDNF in the entire body. This GDNF has been shown to be a potent neurotrophic factor, specifically for motor neurons to modulate neuronal death, and to support neuronal survival and regeneration. Also, production of GDNF has been specifically linked to alpha-2 agonism in cerebral models.

Our next hypothesis moving forward was that treatment of astrocytes with dexmedetomidine, the alpha- 2 agonist, would increase GDNF. We found that astrocytes in general did not produce GDNF on their own. They had to be made to produce it. With dexmedetomidine at $0.1 \mu \mathrm{mol} / \mathrm{L}$, there was a slight increase at 24 hours, although it was not statistically significant. With dexmedetomidine treatment of $1 \mu \mathrm{mol} / \mathrm{L}$ and $10 \mu \mathrm{mol} / \mathrm{L}$, there was a significant increase from all time points compared with control, but specifically 24 hours after treatment with dexmedetomidine, there was a significant increase in GDNF production. By looking further at the $1 \mu \mathrm{mol} / \mathrm{L}$ of dexmedetomidine treatment, we found that at 4, 8, and 16 hours, there was an increasing amount of GDNF. Specifically, at 24 hours we had a higher increase of GDNF and then a drop-off at 36 hours. What this led us to believe is that at 24 hours after dexmedetomidine treatment with $1 \mu \mathrm{mol} / \mathrm{L}$, we were seeing a large increase in GDNF concentration in the astrocyte media. This gave us the idea to look at giving this astrocyte media to the neurons during reperfusion to see if we could elucidate preservation and viability of the neurons. Alpha-2 agonism with dexmedetomidine increases the production of GDNF in both a time- and dose-dependent fashion. Again, we wanted to further investigate what this GDNF would provide as far as neuroprotection.

Our next hypothesis moving forward was that neurons subjected to OGD that are given dexmedetomidine-treated astrocyte media instead of their normal reperfusion media would have a preservation in their viability. Again, this is showing the same part of a graph I have shown before, but just to reiterate, with no OGD we have $100 \%$ viable cells. Down with 100 , or with 1 hour of OGD, we are down to $60 \%$ viable cells. When we added the dexmedetomidine-treated astrocyte media during reperfusion, there was significant preservation and viability up to approximately $80 \%$. This was statistically significant preservation. Again, with 1 hour of OGD with controlled reperfusion media, we have $60 \%$ viable cells. When we use dexmedetomidine-treated astrocyte media instead of reperfusion media, we have up to approximately $80 \%$ viable cells, which is significant preservation.

But what we were wondering was, you know we looked at GDNF. That would be wonderful if that's what was causing the preservation and viability, but how do you know? There could be lots of things in the media that are causing the neurons to be preserved. So what we did was use a GDNF neutralizing antibody, and we found that use of this antibody actually removed the preservation and viability that the astrocyte media gave with the neurons. There was a significant decrease in preservation and viability when we used a neutralizing antibody. What this told us was that specifically, GDNF is causing preservation.

Isolated spinal cord neurons subjected to OGD mimics IR injury. The alpha-2 agonist dexmedetomidine increases GDNF, and dexmedetomidine-treated astrocyte media provides protection from neuronal IR injury via specific GDNF stimulation. Spinal cord cultures can be a powerful tool for future study in spinal cord IR injury, and treatment with the alpha- 2 agonist dexmedetomidine has clinical potential in aortic surgery as a way to prepare the spinal cord for the insult of IR injury.

Dr Ikonimidis (Charleston, SC). Your neutralizing antibody work is compelling with regard to normalization of cell death in this model. I have a few questions related to it. The first is a conceptual one. You acknowledge the fact that you are not able to culture human nerve cells, and so you have to use pediatric cells, pediatric neurons for culture, and clearly this is a disease of the adult spinal cord and not the infant spinal cord. Could you speculate on the potential confounding with regard to the age of the cells?

Dr Freeman. As you know with any in vitro model, you have limitations specific to what works and what does not in vitro, as in certain medias only available, and particularly to neurons. Adult cells just can't be cultured. So in a mouse model, what people do to grow neurons, they use a prenatal mouse, as in cut a pregnant mouse open and use prenatal neurons, or they use postnatal neurons. Now when you talk about low-hanging fruit, culturing prenatal mice neurons is easy. They never die, they always live. Even postnatal mice that are 2 to 3 days old survive approximately $60 \%$ of the time in culture. In adult neurons, although the literature says that it does not really happen, it can't really be done, I tried it anyway, and I got them to stay alive only 2 to 3 days in culture, at best, which is not really long enough for them to settle in.

Dr Ikonimidis. True. Question your ability to separate out cell types. So you are now able to separate out astrocytes from neurons, but the neuronal component is made up of both motor and sensory neurons, and I guess because we are conceptually speaking, we are interested in protecting the motor neurons. Do you have any data on what cell death you are observing in your cultures because the model as you have it has approximately $40 \%$ cell death, which is reduced to $20 \%$ with GDNF. Which neurons are you protecting? Are you protecting motor, sensory, a mix of those? Do you have any comments on that?

Dr Freeman. In general, when you are culturing the spinal cord, you know you can't really separate out. It can't be done with the way that we separate out neurons, and I haven't found a way to separate out them more specifically. If there was a way, that would be great. It would give you even more information on how to preserve the neurons. The only way that we would have knowledge of it would be by morphology, and even then in vitro you can't tell the different cell types. However, there's potential in the future to look toward a way that you can culture out specifically the motor neurons versus nonmotor neurons to get you even closer to identifying what we can do to preserve these.

The GDNF specifically has been shown to be a potent neurotrophic factor for motor neurons, specifically, Parkinson's and amyotrophic lateral sclerosis research has been booming in GDNF. Unfortunately, the biggest issue with GDNF is not whether it's going to help the neurons, but how to deliver it. A lot of the amyotrophic lateral sclerosis literature has been focusing on how to get GDNF to the brain or the cord, and they are having trouble with crossing the blood-brain barrier, which is why dexmedetomidine (Precedex) has such potential because it can elicit this response 
without having to worry about how to get GDNF across the bloodbrain barrier.

Dr Ikonimidis. The astrocyte-conditioned media was delivered to your neurons after 1 hour in your oxygen glucose-deprived state. Clinically, that would equate to a patient developing paraplegia, and then we give the drug. Is that how you envision dexmedetomidine (Precedex) being used clinically? Could you comment on that?

Dr Freeman. I think what we envision for an actual clinical model would be patients admitted the day before, maybe receiving a lumbar drain, things like that, and maybe starting dexmedetomidine at that time. Patients generally tolerate dexmedetomidine (Precedex) very well. We don't know what the dosing would have to be to get the response we see in vitro, but of course that would be sorted out in the future, but giving dexmedetomidine (Precedex) as a pretreatment, I think instead of waiting to see if the patient is one who develops paralysis, that everybody potentially could get it, because the side effect profile is so low.
Dr Ikonimidis. My point being that you might want to consider an experimental design.

Dr Freeman. Absolutely.

Dr Ikonimidis. That includes pretreatment. You're talking about this hypoxia glucose deprivation model as a model of IR, and it probably isn't. Because the definition of ischemia is really a low-flow state, not a low-oxygen state. I think you could give consideration to how you frame your description of that. It might be more of a hypoxia reperfusion model than an IR model. It's semantic, but to basic scientists who do this sort of stuff with oxygendeprived cultures, it's an issue. If you have a lot of volume, you won't develop toxic metabolite accumulation that could contribute to cell death. It's just something to consider.

Dr Freeman. Right. Sometimes we talk about things and it's just semantics, but word choice really is everything when you're describing a model. I think it's the best model we have right now, but there are always ways to improve on what we have.

\title{
EDITORIAL COMMENTARY
}

\section{Do we have a novel, clinically applicable approach to spinal cord protection?}

\author{
John S. Ikonomidis, MD, PhD
}

See related article on pages $578-86$.

Paraplegia remains the most devastating complication related to thoracoabdominal aortic surgery. The early and late presentations may have disparate mechanisms, but nevertheless the outcome is the same, with a profoundly life-changing impact on the patient.

Through the years, numerous strategies have been introduced with the hope of preventing paraplegia, including the use of profound hypothermic circulatory arrest, lumbar drains, intercostal artery reimplantation, and various pharmacologic adjuncts. Indeed, these efforts have shown

From the Division of Cardiothoracic Surgery, Medical University of South Carolina, Charleston, SC.

Disclosures: Author has nothing to disclose with regard to commercial support.

Received for publication Oct 13, 2014; accepted for publication Oct 14, 2014; available ahead of print Nov 7, 2014.

Address for reprints: John S. Ikonomidis, MD, PhD, Division of Cardiothoracic Surgery, Suite 7030, 25 Courtenay Dr, Charleston, SC 29425 (E-mail: ikonomij@musc.edu).

J Thorac Cardiovasc Surg 2015;149:586-7

$0022-5223 / \$ 36.00$

Copyright (c) 2015 by The American Association for Thoracic Surgery

http://dx.doi.org/10.1016/j.jtcvs.2014.10.069 success, and in modern high-volume aortic surgical centers the incidence of paraplegia has dropped below $5 \%$. This devastating complication still looms large, however, for patients undergoing thoracoabdominal aortic surgery.

Recently, various researchers have discovered the ability to "precondition" neurons against damage with pharmacologic agents, some of which seem quite idiosyncratic. The classic example of this is the use of erythropoietin. ${ }^{1}$ Another fascinating and somewhat surprising neuroprotective strategy seems to involve $\alpha_{2}$ adrenergic receptor stimulation. Previously, Bell and colleagues ${ }^{2}$ showed that the $\alpha_{2}$ adrenergic agonist dexmedetomidine, when given postoperatively, attenuates neuronal injury in a murine model of spinal cord ischemia and reperfusion causing delayed paraplegia. The mechanism of action is unclear but may involve increased phosphorylation of protein kinase $B$ and subsequent phosphorylation of cyclic adenosine monophosphate response element-binding protein (CREB), a transcription factor found in previous studies to be critical for neuronal protection and survival. Cyclic adenosine monophosphate response elementbinding protein has been shown to upregulate antiapoptotic factor B-cell lymphoma 2 and brain-derived factors such as glial cell line-derived neurotrophic factor (GDNF), produced by astrocytes. A criticism of the in vivo spinal cord injury 